\title{
ESTUDO GRANULOMÉTRICO DE GRÃOS DE SOJA NORMAL E TRANSGÊNICO
}

\author{
ADHEMAR P. MILANI ${ }^{1}$, VITOR S. BIDÓIA ${ }^{2}$, DILERMANDO PERECIN ${ }^{3}$
}

RESUMO: Este trabalho foi desenvolvido com o propósito de estudar o comportamento granulométrico de grãos de soja em função da variação do teor de água. A pesquisa foi realizada com seis variedades, sendo duas transgênicas e quatro comuns, com os teores de água de $10 \%, 12 \%$, $15 \%, 20 \%$ b.s. A granulometria foi determinada utilizando-se de seis subamostras de 120 grãos para cada teor de água, e as suas dimensões (comprimento, largura e espessura) obtidas por meio de paquímetro digital. Os resultados indicaram que cada variedade de soja comportou-se de maneira diferenciada e que as comuns tiveram comportamento granulométrico diferenciado das transgênicas.

PALAVRAS-CHAVE: granulometria, teor de água, variedades de soja.

\section{NORMAL AND TRANSGENIC SOYBEANS AS A FUNCTION OF THE MOISTURE CONTENT}

\begin{abstract}
This work was developed with the purpose of studying the behavior of soybeans dimensions according to the variation of the moisture content. The research was conducted with six varieties, two transgenic and four normal, with the moisture content: $10 \%, 12 \%, 15 \%$, and $20 \%$ b.s., and the dimensions were determined based on the method used by MILANI et al. (2000). The results indicated that the soybeans varieties showed a differentiated behavior of the dimensions length, width and thickness as a function of moisture content increases, and the normal and transgenic soybeans had different behavior.
\end{abstract}

KEYWORDS: granular dimensions, moisture content, soybeans.

\section{INTRODUÇÃO}

O cultivo de plantas transgênicas, assim como o consumo humano e animal de seus derivados, é um evento recente, revestindo-se de interesses, impactos e conflitos múltiplos, constituindo-se em tema sobre o qual predominam as discussões científicas, éticas, econômicas e políticas nessa transição de século.

A soja vem sendo agregada ao hábito alimentar da população, pois é considerada um produto vegetal de grande concentração proteica. O Brasil posiciona-se como o segundo maior produtor de soja do mundo, perdendo apenas para os Estados Unidos. Nos últimos cinco anos, a produção de soja no País quase dobrou; no ano de 2006, a produção de soja em grãos foi de 52.355 .976 toneladas, e para 2007 houve aumento significativo de 11,22\%.

As exportações vêm firmando-se nos últimos anos, garantindo e mantendo como exportador regular, sendo a soja o produto mais plantado no País (FAO, 2008; IBGE, 2007), resultando na necessidade de aumentar a capacidade de armazenamento. Nesse sentido, o País ainda apresenta problemas quanto ao armazenamento de grãos, sendo comum, em algumas regiões, a perda de parte das colheitas devido à insuficiência da capacidade de armazenamento ou mau armazenamento, demonstrando a necessidade de desenvolver estudos e investimentos nesse setor.

\footnotetext{
${ }^{1}$ Eng ${ }^{0}$ Civil, Prof. Dr., Departamento de Engenharia Rural, UNESP, Câmpus de Jaboticabal - SP, Fone: (0xx16) 3209.2637, apmilani@fcav.unesp.br

${ }^{2}$ Estudante de graduação, Departamento de Engenharia Rural, UNESP, Câmpus de Jaboticabal - SP, Fone: (0xx16) 3209.2637.

${ }^{3}$ Eng ${ }^{0}$ Agrônomo, Prof. Titular, Departamento de Ciências Exatas, UNESP, Câmpus de Jaboticabal - SP, Fone: (0xx16) 3209.2624.

Recebido pelo Conselho Editorial em: 16-12-2008

Aprovado pelo Conselho Editorial em: 23-11-2009 
Das propriedades físicas dos grãos, o conhecimento da granulometria, associado ao teor de água, é de suma importância no projeto de silos considerado pelas principais normas internacionais (ACI, 1997; ASAE, 2000a; ASAE, 2000b; DIN, 1987; NRCC, 1995; STANDARDS AUSTRALIA, 1996), bem como na definição dos parâmetros a serem adotados na regulagem de implementos agrícolas, como colhedoras e plantadoras. Com o advento do desenvolvimento tecnológico, muitas variedades de sojas são disponibilizadas no mercado ao ano, principalmente as transgênicas, e há poucas informações a respeito da sua granulometria em relação ao teor de água.

Assim sendo, este trabalho teve como objetivo o estudo do comportamento granulométrico de grãos de soja transgênico e comum, em função do teor de água.

\section{MATERIAL E MÉTODOS}

O trabalho foi realizado no Departamento de Engenharia Rural, da Faculdade de Ciências Agrárias e Veterinárias, UNESP-Câmpus de Jaboticabal. Nos estudos, foram utilizadas seis variedades de soja, sendo quatro normais: Conquista, Monsoy M-SOY 8001, Coodetec 2006 e Semente SOL-CD 216, e duas transgênicas: BRS Valiosa RR e MG/BR 46.

Primeiramente, determinou-se o teor de água inicial dos grãos, por meio do método da estufa, utilizando-se de três amostras de $15 \mathrm{~g}$, à temperatura de $103{ }^{\circ} \mathrm{C}$, com tolerância de $\pm 1{ }^{\circ} \mathrm{C}$, por um período de 72 h. Após essa determinação, efetuou-se a correção do teor de água dos grãos (10; 12; 15 ; e $20 \%$ b.s.), calculados por meio das seguintes equações:

$$
\begin{aligned}
& \mathrm{Ps}=\frac{P u i}{U+1} \\
& \mathrm{Pu}-P s(U+1) \\
& \mathrm{Qa}=\mathrm{Pu}-\mathrm{P}_{u i}
\end{aligned}
$$

em que,

Pui - peso úmido inicial, $1.000 \mathrm{~g}$;

Ps - peso seco, g;

$\mathrm{U}$ - teor de água, \%, e

$\mathrm{Qa}$ - quantidade de água, $\mathrm{g}$.

Em seguida, os grãos eram colocados em sacos plásticos, adicionava-se água (destilada) determinada e armazenava-se por um período de quatro dias em geladeira, à temperatura em torno de $5{ }^{\circ} \mathrm{C}$, para uniformização da massa de grãos. A determinação da granulometria dos grãos foi realizada com base em MILANI et al. (2000), utilizando-se de seis subamostras de 120 grãos para cada teor de água. A obtenção das dimensões dos grãos (comprimento, largura e espessura) foi feita por meio de paquímetro digital. Os resultados obtidos foram avaliados por meio de análise de variância linear e análise fatorial.

\section{RESULTADOS E DISCUSSÃO}

Nas Tabelas 1 e 2, apresentam-se os valores médios das dimensões dos grãos de soja normal e trangênico, respectivamente, em função do teor de água. Dos resultados, verifica-se que os grãos apresentaram comportamentos diferentes de suas dimensões (comprimento, largura e espessura) ao incremento do teor de água. 
TABELA 1. Valores médios das dimensões dos grãos de soja normal (comprimento-C, largura-L e espessura-E) em função do teor de água. Dimension mean values of normal soybeans (length-C, width-L and thickness-E) in function of the water content.

\begin{tabular}{ccccccccccccc}
\hline \multirow{2}{*}{$\begin{array}{c}\text { Teor de } \\
\text { Água (\%) }\end{array}$} & \multicolumn{4}{c}{ Conquista } & \multicolumn{1}{c}{ M-SOY 8001 } & \multicolumn{3}{c}{ Coodetec 2006 } & \multicolumn{3}{c}{ SOL -CD 216 } \\
\cline { 2 - 13 } & C & L & E & C & L & E & C & L & E & C & L & E \\
\hline 10 & 7,36 & 6,58 & 5,46 & 6,63 & 5,97 & 5,19 & 7,44 & 6,02 & 4,54 & 7,00 & 5,64 & 4,48 \\
12 & 7,38 & 6,77 & 5,79 & 6,57 & 5,84 & 5,13 & 7,50 & 6,03 & 4,51 & 6,97 & 5,60 & 4,45 \\
15 & 7,50 & 6,79 & 5,88 & 6,67 & 5,87 & 5,14 & 7,63 & 6,11 & 4,56 & 7,08 & 5,67 & 4,45 \\
20 & 8,00 & 6,95 & 5,86 & 7,04 & 5,99 & 5,24 & 8,01 & 6,24 & 4,56 & 7,46 & 5,71 & 4,44 \\
\hline
\end{tabular}

TABELA 2. Valores médios das dimensões dos grãos de soja transgênica (comprimento-C, larguraL e espessura-E) em função do teor de água. Dimension mean values of transgenic soybeans (length-C, width-L and thickness-E) in function of the water content.

\begin{tabular}{ccccccc}
\hline \multirow{2}{*}{$\begin{array}{c}\text { Teor de Água } \\
(\%)\end{array}$} & \multicolumn{7}{c}{ BRS Valiosa RR } \\
\cline { 2 - 7 } & C & L & E & C & L & E \\
\hline 10 & 6,93 & 6,50 & 5,55 & 7,36 & 6,58 & 5,46 \\
12 & 7,18 & 6,63 & 5,65 & 7,35 & 6,50 & 5,38 \\
15 & 7,22 & 6,66 & 5,69 & 7,53 & 6,64 & 5,52 \\
20 & 7,45 & 6,78 & 5,74 & 7,83 & 6,63 & 5,56 \\
\hline
\end{tabular}

Na Tabela 3, são apresentados os resultados obtidos das médias gerais das dimensões (mm): comprimento, largura e espessura, para a variável teor de água (\%), por meio do teste de Tukey, demonstrando seus comportamentos. Evidencia-se que, para o comprimento e para a largura, há diferença significativa entre os teores de água de $20 \%$ para $15 \%$, que se diferem dos de 12 e $10 \%$, sendo o comportamento dessas duas últimas semelhantes com relação à variação de suas dimensões.

Já para o comportamento da espessura, ocorre que, no teor de água de $20 \%$, acontece comportamento semelhante ao teor de água de $15 \%$, sendo que ambos diferem para $12 \%$, que, por sua vez, difere-se de $10 \%$, que apresenta comportamento diferenciado das demais.

TABELA 3. Valores das médias gerais do teor de água (\%) para as dimensões comprimento, largura e espessura. General water content averages (\%) for length, width and thickness.

\begin{tabular}{cccc}
\hline \multirow{2}{*}{ Teores de Água $(\%)$} & \multicolumn{3}{c}{ Médias Gerais das Dimensões $(\mathrm{mm})$} \\
\cline { 2 - 4 } & Comprimento & Largura & Espessura \\
\hline 20 & $7,63 \mathrm{~A}$ & $6,38 \mathrm{~A}$ & $5,23 \mathrm{~A}$ \\
15 & $7,27 \mathrm{~B}$ & $6,29 \mathrm{~B}$ & $5,21 \mathrm{~A}$ \\
12 & $7,16 \mathrm{C}$ & $6,23 \mathrm{C}$ & $5,15 \mathrm{~B}$ \\
10 & $7,12 \mathrm{C}$ & $6,22 \mathrm{C}$ & $5,11 \mathrm{C}$ \\
\hline
\end{tabular}

Obs: letras diferentes indicam significância $(\mathrm{P}<0,05)$ pelo teste de Tukey.

$\mathrm{Na}$ Tabela 4, são evidenciados valores das médias gerais das dimensões comprimento, largura e espessura, com relação ao comportamento dentro de cada variedade. Assim, observa-se pelo teste de Tukey que cada variedade de soja se comporta de maneira diferente em função do teor de água médio, diferenciando-se umas das outras. Para comprimento, a variedade Coodetec 2006 destaca-se com os maiores valores médios, ocorrendo dessa forma destaque para largura e espessura para Conquista. 
TABELA 4. Resultados do teste de Tukey para os valores das médias gerais de variedades de soja, para as dimensões comprimento, largura e espessura. Tukey test results for the general average values of soybean varieties for length, width and thickness.

\begin{tabular}{ccccc}
\hline \multirow{2}{*}{ Classe } & \multirow{2}{*}{ Variedades } & \multicolumn{3}{c}{ Médias Gerais das Dimensões (mm) } \\
\cline { 2 - 5 } & & Comprimento & Largura & Espessura \\
\hline Normal & Coodetec 2006 & $7,64 \mathrm{~A}$ & $6,10 \mathrm{D}$ & $4,54 \mathrm{E}$ \\
Normal & Conquista & $7,56 \mathrm{~B}$ & $6,77 \mathrm{~A}$ & $5,75 \mathrm{~A}$ \\
Transgênica & MG/BR 46 & $7,52 \mathrm{~B}$ & $6,59 \mathrm{C}$ & $5,48 \mathrm{C}$ \\
Transgênica & BRS Valiosa RR & $7,20 \mathrm{C}$ & $6,64 \mathrm{~B}$ & $5,66 \mathrm{~B}$ \\
Normal & Semente Sol - CD 216 & $7,13 \mathrm{D}$ & $5,66 \mathrm{~F}$ & $4,46 \mathrm{~F}$ \\
Normal & Monsoy M-SOY 8001 & $6,73 \mathrm{E}$ & $5,92 \mathrm{E}$ & $5,17 \mathrm{D}$ \\
\hline
\end{tabular}

Na Tabela 5, são apresentados os valores gerais da análise de variância para comprimento, largura e espessura, e suas variáveis: umidade, classe (normal ou transgênica) e variedade dentro de sua respectiva classe. Também estão presentes seus respectivos coeficientes de variação, graus de liberdade e valores médios das dimensões. Assim, pode-se dizer que há diferença significativa para os itens comprimento, largura e espessura, nos quesitos umidade, classe e variedade. Verifica-se que, com o incremento dos teores de água presentes nos grãos, ocorre variação significativa nas dimensões dos grãos entre si, ocorrendo, também, diferenciação significante no comportamento da soja normal e transgênica, e cada variedade dentro de sua classe também sofreu diferenciação significativa, demonstrando que cada variedade comportou-se de forma diferente. Seus respectivos coeficientes de variação apresentaram-se baixos, evidenciando a credibilidade na veracidade dos dados obtidos.

TABELA 5. Quadrado médio, grau de liberdade (GL), análise de variância, média geral e coeficientes de variação para as dimensões comprimento, largura e espessura. Mean square, freedom degree (GL), variance analysis, mean average and variation coefficients for length, width and thickness.

\begin{tabular}{ccccc}
\hline Fatores de Variação & GL & Comprimento & Largura & Espessura \\
\hline Teor de água & 3 & $234,52^{* *}$ & $25,35^{* *}$ & $12,88^{* *}$ \\
Variedades & $(5)$ & & & \\
Normais vs Transgênicas & 1 & $31,78^{* *}$ & $972,40^{* *}$ & $1.339,53^{* *}$ \\
Entre normais & 3 & $516,92^{* *}$ & $653,61^{* *}$ & $1.050,23^{* *}$ \\
Entre transgênicas & 1 & $148,10^{* *}$ & $4,11^{* *}$ & $45,87^{* *}$ \\
Teor de água x variedades & 15 & $2,99 * *$ & $3,26^{* *}$ & $5,27^{* *}$ \\
Resíduo & 17.271 & 0,816 & 0,089 & 0,437 \\
\hline C.V.\% & - & 12,38 & 4,76 & 12,77 \\
\hline Média Geral & - & 7,29 & 6,28 & 5,17 \\
\hline
\end{tabular}

*para 5\% de significância, ** para 1\% de significância.

Embora os quadrados médios da interação "teor de água x variedades" sejam de magnitude relativa baixa, tanto em relação ao teor de água como em relação à de variedades, os mesmos são significativos, ou seja, há diferenças entre variedades quanto à resposta ao teor de água. Para tanto, na Tabela 6, mostram-se as taxas de aumento por unidade de teor de água, complementando as informações dos dados das Tabelas 1 e 2.

Embora haja diferenças nas taxas de expansão entre as variedades, as maiores expansões ocorrem com a variedade normal Conquista. 
TABELA 6. Taxa de aumento ou de expansão por unidade de teor de água. Increase or expansion rate per unit of water content.

\begin{tabular}{ccccc}
\hline \multirow{2}{*}{ Classe } & \multirow{2}{*}{ Variedades } & \multicolumn{3}{c}{ Taxa (mm) } \\
\cline { 3 - 5 } & MG/BR 46 & 0,0500 & Largura & Espessura \\
\hline Transgênica & MRrimento & 0,0090 & 0,0142 \\
Transgênica & BR Valiosa RR & 0,0469 & 0,0257 & 0,0170 \\
Normal & Conquista & 0,0658 & 0,0324 & 0,0333 \\
Normal & Coodetec 2006 & 0,0577 & 0,0231 & 0,0035 \\
Normal & Semente Sol - CD 216 & 0,0492 & 0,0092 & $-0,0032$ \\
Normal & Monsoy M-SOY 8001 & 0,0444 & 0,0056 & 0,0068 \\
\hline
\end{tabular}

\section{CONCLUSÕES}

Os resultados obtidos no presente trabalho evidenciaram que as variedades de soja apresentaram comportamento diferenciado de suas dimensões com relação aos teores de água, e que as sojas normal e transgênica apresentam comportamentos granulométricos diferentes.

\section{AGRADECIMENTOS}

À Empresa Brasileira de Pesquisa Agropecuária - Embrapa, pelo fornecimento do material transgênico.

\section{REFERÊNCIAS}

ACI. Standard practice for design and construction of concrete silos and stacking tubes for storing granular materials and commentary. Detroit: American Concrete Institute. 1997. (ACI 313r-97)

ASAE Standards. Loads exerted by free-flowing grain on bins. St. Joseph, 2000a. ANSI/ASAE EP433

\section{EP545.}

. Loads exerted by free-flowing grains on shallow storage. St. Joseph, 2000b. ANSI/ASAE

DIN. Lastnnahmen fur bautten- en lasten in silozellen (Design loads for buildings-Loads in silos).

Deutsche, 1987. (DIN 1055, Part 6)

IBGE. INSTITUTO BRASILEIRO DE GEOGRAFIA E ESTATÍSTICA. Levantamento

Sistemático da produção agrícola. Disponível em:

<http://www1.ibge.gov.br/home/estatistica/indicadores/agropecuaria/lspa/lspa_200707_5.shtm>. Acesso em: 29 ago. 2007

FAO. FOOD AND AGRICULTURE ORGANIZATION OF THE UNITED NATIONS. Disponível em: <www.fao.org>. Acesso em: 10 fev. 2008.

MILANI, A.P.; BUCKLIN, R.A.; TEIXEIRA, A.A.; KEBELL, H.V. Soybean compressibility and bulk density. Transactions of the ASAE, St. Joseph, v.43, n.6, p.1.789-1.793, 2000.

NRCC. NATIONAL FARM BUILDING CODE OF CANADÁ. Canadian Commission on Building and Fire Codes. Ottawa: National Research Council of Canada, 1995. (NRCC 38732)

STANDARDS AUSTRALIA. Loads on bulk solids containers. Homebush, Austrália. 1996. (AS 3774-1996) 